\title{
Multidetector-computed tomography attenuation values between the tumor and aortic wall in response to induction therapy for esophageal cancer and its predictive value for aortic invasion
}

\author{
HIRONORI TSUJIMOTO, TAKASHI ICHIKURA, SATOSHI AIKO, YOSHIHISA YAGUCHI, \\ ISAO KUMANO, RISA TAKAHATA, YUSUKE MATSUMOTO, KAZUMICHI YOSHIDA, \\ SATOSHI ONO, JUNJI YAMAMOTO and KAZUO HASE
}

Department of Surgery, National Defense Medical College, Tokorozawa 359-8513, Japan

Received August 24, 2011; Accepted October 3, 2011

DOI: 10.3892/etm.2011.386

\begin{abstract}
The objective of this study was to evaluate the multidetector computed tomography (MDCT) attenuation value between the tumor and aorta in response to the induction therapy for esophageal cancer. In advanced esophageal cancer, the main reason for unresectability is the local invasion of the tumor into the aorta or trachea. Despite remarkable advances in diagnostic modalities, pre-operative assessment of pathological response and local tumor extent in esophageal cancer remains difficult. MDCT attenuation values between the tumor and aorta, and the contact angle of the tumor to the aorta (Picus' angle) were retrospectively evaluated in patients with esophageal cancer who underwent induction therapy in terms of predicting the pathological response, aortic invasion and prognosis of esophageal cancer. The induction therapy may increase the tumor-to-aorta distance and decrease the maximum tumor size and Picus' angle. When the tumor-to-aorta cut-off value was set at $<1.3 \mathrm{~mm}$, the accuracy of this distance for aortic invasion was $94.6 \%$. In terms of this distance, 14 out of 19 patients with a tumor-to-aorta distance of $<1.3 \mathrm{~mm}$ prior to the induction therapy had a distance of $>1.3 \mathrm{~mm}$ following therapy and underwent curative resection. The assessment of the MDCT attenuation value between the esophageal tumor and the aorta is simple and objectively assesses the response to the induction therapy and aortic invasion in esophageal cancer. This method should be applied to predict the response to the induction therapy and to prevent unnecessary surgery in patients with tumors involving the aorta.
\end{abstract}

Correspondence to: Dr Hironori Tsujimoto, Department of Surgery, National Defense Medical College, 3-2 Namiki, Tokorozawa 359-8513, Japan

E-mail: tsujihi@ndmc.ac.jp

Key words: multidetector computed tomography, induction therapy, esophageal cancer, cancer imaging, aortic invasion, response rate

\section{Introduction}

The optimal treatment of squamous cell carcinoma of the esophagus remains to be elucidated $(1,2)$. As the esophagus is not surrounded by a serosal lining, infiltration into adjacent mediastinal structures, including the aorta, tracheobronchial tree and vertebral column, occurs easily (3). It is extremely important to accurately evaluate tumor invasion of the adjacent mediastinal structures in order to prevent unnecessary surgery in patients with inoperable tumors $(4,5)$.

At the time of diagnosis, fewer than half the patients have locally advanced tumors that are resectable (6). In these patients, induction therapies, such as pre-operative chemotherapy and chemoradiotherapy, offer a survival advantage compared to surgery alone (7). However, in patients who respond unfavorably, the inefficient induction therapy should be discontinued and surgery should not be delayed. On the other hand, patients who respond favorably to the induction therapy may benefit from additional pre-operative treatment. Thus, there is a need for a method that may be used to reliably predict the pathological response to the induction therapy in order to prevent wastage of time and unnecessary surgery.

Currently, endoscopic ultrasound (EUS) is used to assess the local tumor extent in esophageal cancer $(8,9)$; unfortunately, many patients with locally advanced cancer have too narrow a lumen to allow passage of the endoscope (3). Alternatively, prediction of aortic invasion has also been evaluated with computed tomography (CT). The overall circumference of contact between the tumor and the aortic wall has been shown to be a useful predictor, with an interface arc greater than 90 degrees, suggesting invasion, as reported by Picus et al (10). Currently used multidetector CT (MDCT) scanners enable thinner collimation and faster scanning, which markedly improves imaging resolution and enables rapid handling of image reconstruction (11). Therefore, we hypothesized that the evaluation of intervening tissues between the tumor and aortic wall visualized by MDCT may enable assessment of the induction therapy response and aortic invasion.

In the present study, we retrospectively evaluated MDCT attenuation values between the tumor and aortic wall prior to and following the induction therapy, and examined whether 
attenuation values could be used to assess aortic invasion in patients with advanced esophageal cancer.

\section{Materials and methods}

Patients. A total of 162 consecutive patients underwent transthoracic esophagectomy for esophageal cancer at the National Defense Medical College Hospital (Japan) from January 2005 to May 2010. Out of 162 patients, 56 who were suspected to have a tumor invading the adventitia (T3) without any distant metastasis underwent an induction therapy, chemotherapy or chemoradiotherapy, and were enrolled in this study. The pathological and clinical stages of the tumors in these patients were determined according to the 5th edition of the tumor-nodemetastasis (TNM) Classification of Malignant Tumors of the International Union Against Cancer (12). Pathological criteria for the effects of chemotherapy or chemoradiotherapy are described based on the Japanese Classification of Esophageal Cancer, 10th edition (13): grade 1a, viable cancer cells account for two thirds or more of the tumor tissue, but there is some evidence of degeneration of cancer tissue or cells; grade $1 b$, viable cancer cells account for one third or more of the tumor tissue, but less than two thirds of tumor tissue; grade 2, viable cancer cells account for less than one third of the tumor tissue, while other cancer cells are severely degenerated or necrotic. Surgical findings regarding the extension of the tumor were described according to the medical records. All surgeries were performed by three experts who had more than 10 years of experience in performing esophagectomies (H.T., T.I. and S.A.).

The chemotherapeutic regimens included two courses of chemotherapy of cisplatin (CDDP; $80 \mathrm{mg} / \mathrm{m}^{2}$ of intravenous drip infusion, day 1) and 5-fluorouracil (5-FU; $800 \mathrm{mg} / \mathrm{m}^{2}$ of continuous infusion, days 1-5). For the chemoradiotherapy, the chemotherapeutic regimens included the administration of CDDP (70 mg/m² of intravenous drip infusion, day 1) and 5 -FU (700 $\mathrm{mg} / \mathrm{m}^{2}$ of continuous infusion, days $\left.1-4\right)$, and the concurrent radiation therapy was planned to be administered in daily fractions of 2 Gy for a total dose of 30-40 Gy (median 30.9). The chemotherapy or chemoradiotherapy as an induction therapy was chosen, taking risk and benefit into consideration. Written informed consent was obtained from all individuals prior to initiation of the study.

Evaluation of CT attenuation values between the tumor and aorta. All studies were performed using a 64-detector row CT (Aquilion $^{\text {TM }}$ 64; Toshiba Medical, Tokyo, Japan). A total of $300 \mathrm{ml}$ of non-ionic contrast agents were intravenously administered at a speed of $3 \mathrm{ml} / \mathrm{sec}$, and CT was performed $120 \mathrm{sec}$ following injection. To adjust the $\mathrm{CT}$ attenuation values, water and air calibrations were performed quarterly and weekly, respectively. The scanning parameters included $120 \mathrm{kVp}$, 0.5 -sec tube rotation time, $27 \mathrm{~mm} /$ rotation helical pitch, $55 \mathrm{~mm}$ table speed, 0.5 -sec gantry rotation time and $2.5-\mathrm{mm}$ thick reconstructed sections. The images were reviewed on a workstation (Zio workstation; AMIN Inc., Tokyo, Japan). These were independently and retrospectively evaluated by two surgeons who had more than 10 years of experience in performing esophagectomies and were blinded to complaints, specific medical history and findings of physical examination, surgery, laboratory evaluation and imaging.
The maximum tumor size in a horizontal section, tumor location and CT attenuation value before and after induction therapies were described. The overall circumference of contact between the tumor and aortic wall (Picus' angle) was determined, as previously described (10). To determine the CT attenuation value between the tumor and aorta, consecutive CT values between the center of the tumor and the center of the aorta were determined using a Zio workstation. We examined the average CT attenuation value of the tumor and determined the distance between the intersections of this average with the lower $\mathrm{CT}$ attenuation value of the inclusion tissues (Fig. 1). In our previous examinations, which included 101 patients with advanced esophageal cancer, serial cut-off values were inserted around the inflection points on the receiver operating characteristic (ROC) curve for the pathological aortic invasion of the esophageal cancer (Fig. 1). We also determined the minimum $\mathrm{CT}$ attenuation value between the tumor and aorta.

Statistical analysis. The data are expressed as the means \pm standard deviation (SD). Comparison between the two groups was analyzed using the Mann-Whitney U test or Wilcoxon signed rank test. These data were analyzed using MedCalc version 9 statistical software package (MedCalc software, Mariakerke, Belgium). A p-value of $<0.05$ was considered to be statistically significant.

\section{Results}

The demographic data of the patients are presented in Table I. There were no differences in the distance and minimum CT value between the tumor and aorta, and Picus' angle due to age, gender and body mass index (data not shown). The potentially curative resections were achieved in $78.6 \%$. A total of 27 patients received pre-operative chemotherapy and 29 patients received pre-operative chemoradiotherapy. A total of 7 patients $(12.5 \%)$ were suspected to have tumor invasion of the aortic wall during surgery, and 6 patients (10.7\%) were pathologically confirmed to have tumor invasion of the aortic wall. The result of the pathological response to the induction therapy was 39 patients (chemotherapy:chemoradiotherapy, $23: 16)$ with grade 1 a disease, 6 patients $(2: 4)$ with grade $1 \mathrm{~b}$ disease and 11 patients (2:9) with grade 2 disease.

The tumor-to-aorta distance following induction therapy was significantly higher than that prior to induction therapy $(2.4 \pm 1.1$ vs. $1.8 \pm 0.9 \mathrm{~mm})$. The maximum tumor sizes and Picus' angles following the induction therapy were significantly reduced compared to those prior to the induction therapy $(30.5 \pm 9.9$ vs. $34.8 \pm 8.6 \mathrm{~mm}$, and $68.9 \pm 31.6$ vs. $75.0 \pm 25.6$ degrees, respectively). However, there were no differences in the minimum CT attenuation value between the tumor and aorta prior to and following the induction therapy (65.4 \pm 32.3 vs. $61.4 \pm 29.9$ Hounsfield units).

We compared each parameter before and after the induction therapy according to the pathological response (Fig. 2). There were significant differences in the tumor-to-aorta distance and maximum tumor size among the pathological responses, whereas such differences were not observed in the Picus' angles and minimum CT attenuation values between the tumor and aorta. We then compared each parameter prior to 



Figure 1. CT attenuation curves between the center of the tumor and the center of the aorta. The average CT attenuation value of the tumor was examined and the distance between the intersections of this value, with the lower CT attenuation value of the connective tissue, was determined. The minimum CT attenuation value between the tumor and the aorta was also determined. T, tumor; A, aorta; S, spine; $\mathrm{CT}$, computed tomography.

and following the induction therapy according to the therapeutic approach, such as chemotherapy and chemoradiotherapy (Table I). The tumor-to-aorta distance following the induction therapy was significantly higher than that prior to the induction therapy in patients who underwent chemotherapy, while Picus' angle following the induction therapy was significantly reduced compared to that prior to the induction therapy in patients who underwent chemoradiotherapy. The maximum tumor size was significantly reduced following both induction therapies, and we were unable to find any differences in the minimum $\mathrm{CT}$ attenuation value before and after the induction therapy (Table II).

Since 6 patients were pathologically confirmed to have tumor invasion of the aortic wall, we compared each parameter between patients with and without aortic invasion (Fig. 3). There were significant differences in the tumor-to-aorta distance, maximum tumor size and Picus' angle before and after the induction therapy in patients without aortic invasion; however, such differences were not observed in patients with aortic invasion and in the minimum $\mathrm{CT}$ attenuation value between the tumor and aorta prior to and following the induction therapy. Notably, in patients with aortic invasion, the tumor-to-aorta distance following the induction therapy was shorter than that prior to the induction therapy, albeit not significantly. Furthermore, there were significant differences following the induction therapy in the distance and minimum
Table I. Demographic data of patients who were suspected to have a tumor invading the adventitia (T3) without any distant metastasis.

\begin{tabular}{|c|c|}
\hline Characteristic & No. of patients $(n=56)$ \\
\hline Age (years) & $64.3 \pm 7.5$ \\
\hline \multicolumn{2}{|l|}{ Gender } \\
\hline Male & 52 \\
\hline Female & 4 \\
\hline \multicolumn{2}{|l|}{ Location } \\
\hline Upper thoracic & 4 \\
\hline Middle thoracic & 27 \\
\hline Lower thoracic & 15 \\
\hline Abdominal esophagus & 10 \\
\hline \multicolumn{2}{|l|}{ Histology } \\
\hline SCC & 51 \\
\hline Well & 4 \\
\hline Moderate & 41 \\
\hline Poor & 8 \\
\hline Adenocarcinoma & 3 \\
\hline \multicolumn{2}{|l|}{ Tumor depth } \\
\hline pT2 & 6 \\
\hline pT3 & 40 \\
\hline pT4 & 10 \\
\hline Aorta & 6 \\
\hline Lung & 2 \\
\hline Trachea & 1 \\
\hline Pericardiac membrane & 1 \\
\hline \multicolumn{2}{|l|}{ Lymphadenectomy } \\
\hline 2-field & 24 \\
\hline 3-field & 32 \\
\hline \multicolumn{2}{|l|}{ Residual tumor } \\
\hline R0 & 44 \\
\hline $\mathrm{R} 1$ & 5 \\
\hline $\mathrm{R} 2$ & 7 \\
\hline \multicolumn{2}{|l|}{ Induction therapy } \\
\hline Chemotherapy & 27 \\
\hline Chemoradiation therapy & 29 \\
\hline \multicolumn{2}{|l|}{ Pathological response } \\
\hline Grade 1a & 39 \\
\hline Grade $1 \mathrm{~b}$ & 6 \\
\hline Grade 2 & 11 \\
\hline \multicolumn{2}{|l|}{ Aortic invasion } \\
\hline \multicolumn{2}{|l|}{ Surgical } \\
\hline Yes & 7 \\
\hline No & 49 \\
\hline \multicolumn{2}{|l|}{ Pathological } \\
\hline Yes & 6 \\
\hline No & 50 \\
\hline
\end{tabular}

SCC, squamous cell carcinoma.

CT attenuation values between the tumor and aorta, and Picus' angles between patients with and without aortic invasion; however, this was not observed in the maximum tumor size. 

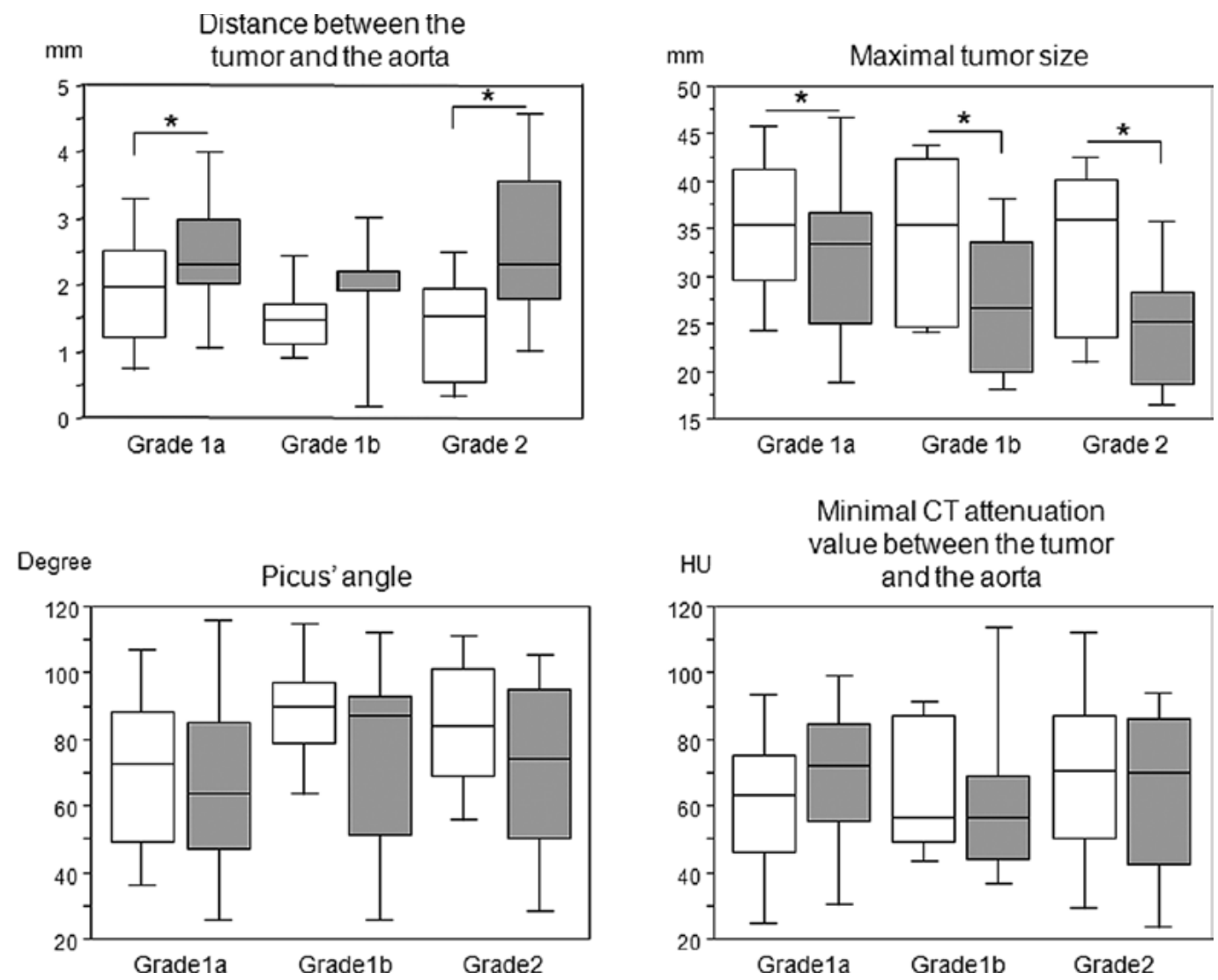

Figure 2. Distance and minimum CT attenuation value between the tumor and aorta, Picus' angle and maximum tumor size according to the pathological response to the induction therapy. Open square, prior to the induction therapy; Gray-filled square, following the induction therapy. * $<<0.05$ calculated by the Wilcoxon signed rank test. CT, computed tomography.
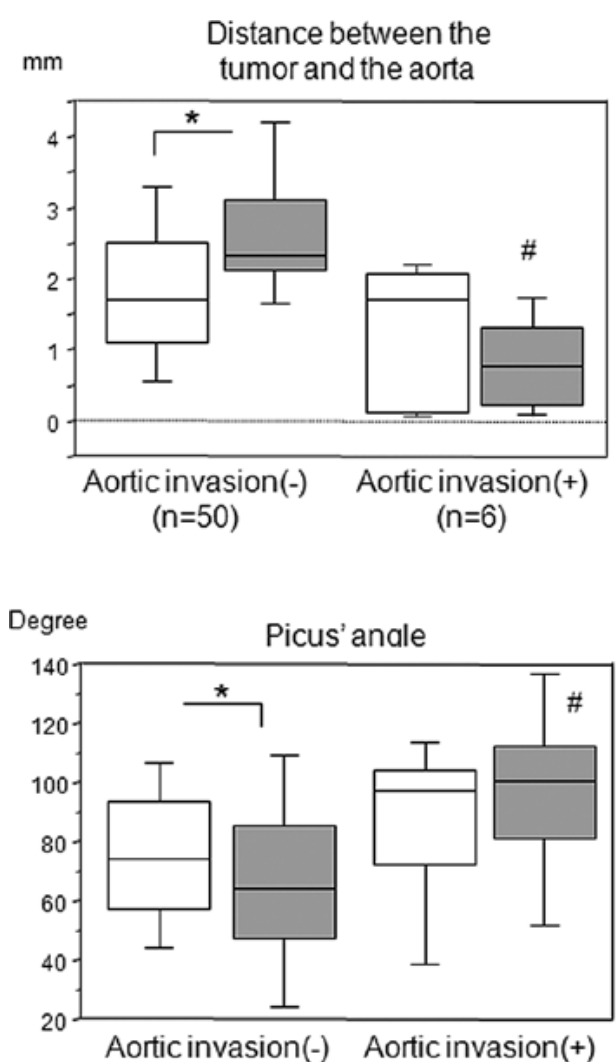

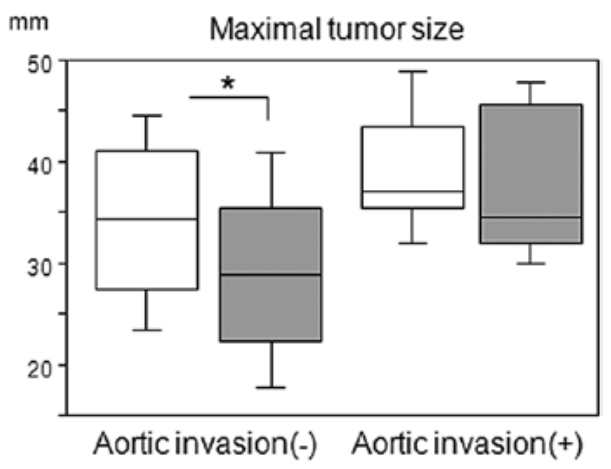

Minimal CT attenuation value between the tumor

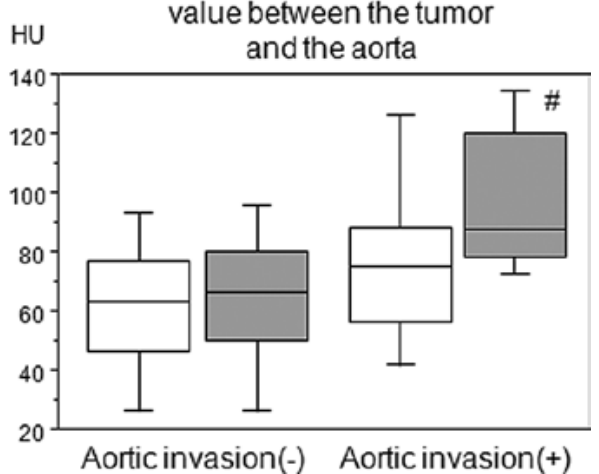

Figure 3. Tumor-to-aorta distance, minimum CT attenuation value between the tumor and aorta, Picus' angle and maximum tumor size in patients with or without aortic invasion. Open square, prior to the induction therapy; Gray-filled square, following the induction therapy. * $<<0.05$ calculated by the Wilcoxon signed rank test. ${ }^{\#} \mathrm{p}<0.05$ vs. without aortic invasion following induction therapy, calculated by the Mann-Whitney U test. CT, computed tomography. 
Table II. Distance and minimum CT attenuation value between the tumor and aorta, Picus' angle and maximum tumor size before and after the induction therapy.

\begin{tabular}{lccc}
\hline & Before & After & p-value \\
\hline Distance & & & \\
Chemotherapy & $1.9 \pm 0.9$ & $2.8 \pm 1.2$ & 0.0001 \\
Chemoradiotherapy & $1.7 \pm 1.0$ & $2.1 \pm 1.0$ & 0.0846 \\
Total & $1.8 \pm 0.9$ & $2.4 \pm 1.1$ & 0.0001 \\
Minimal CT & & & \\
Chemotherapy & $53.2 \pm 33.6$ & $56.6 \pm 36.3$ & 0.9291 \\
Chemoradiotherapy & $69.7 \pm 23.6$ & $73.7 \pm 26.2$ & 0.5903 \\
Total & $61.4 \pm 29.9$ & $65.4 \pm 32.3$ & 0.6594 \\
Picus' angle & & & \\
Chemotherapy & $65.9 \pm 25.0$ & $63.3 \pm 32.9$ & 0.2795 \\
Chemoradiotherapy & $84.1 \pm 23.2$ & $74.0 \pm 30.0$ & 0.0044 \\
Total & $75.0 \pm 25.6$ & $68.9 \pm 31.6$ & 0.0049 \\
Maximal tumor size & & & \\
Chemotherapy & $34.2 \pm 7.8$ & $29.7 \pm 9.6$ & 0.0014 \\
Chemoradiotherapy & $35.4 \pm 9.5$ & $31.2 \pm 10.3$ & 0.0003 \\
Total & $34.8 \pm 8.6$ & $30.5 \pm 9.9$ & 0.0001 \\
\hline
\end{tabular}

Distance, the distance between the tumor and the aorta; minimum $\mathrm{CT}$, the minimum $\mathrm{CT}$ attenuation value between the tumor and aorta. Calculated by the Wilcoxon signed rank test.

In our previous examinations, which included 101 patients with advanced esophageal cancer, when the cut-off value of the tumor-to-aorta distance was set at $<1.3 \mathrm{~mm}$, the sensitivity, specificity and accuracy for the pathological aortic invasion were $87.5,91.4$ and $91.1 \%$, respectively (Fig. 1). A total of 19 patients had a tumor-to-aorta distance of $<1.3 \mathrm{~mm}$ prior to the induction therapy, and in 14 out of the 19 patients, the tumor-to-aorta distance increased to $>1.3 \mathrm{~mm}$ following the induction therapy; none of these patients had aortic invasion (Fig. 4). The remaining 5 patients continued to have a tumor-toaorta distances of $<1.3 \mathrm{~mm}$ following the induction therapy; 4 of these 5 patients had aortic invasion. However, 2 out of 37 patients in whom tumor-to-aorta distance was $>1.3 \mathrm{~mm}$ prior to the induction therapy had a decrease in this distance $(<1.3 \mathrm{~mm})$ following the induction therapy; the two patients had aortic invasion and a grade la pathological response.

\section{Discussion}

In the present study, we evaluated the $\mathrm{CT}$ attenuation value between the tumor and aorta in response to the induction therapy for advanced esophageal cancer using MDCT. We demonstrated that the tumor-to-aorta distance and maximum tumor size reflect the pathological response to the induction therapy. In addition, the induction therapy may increase the tumor-to-aorta distance, and decrease the maximum tumor size and Picus' angle in patients with esophageal cancer.

Conventionally, CT scans and 18-F-fluorodeoxyglucose positron emission tomography have been widely employed to evaluate the tumor response to chemotherapy and/or radiation

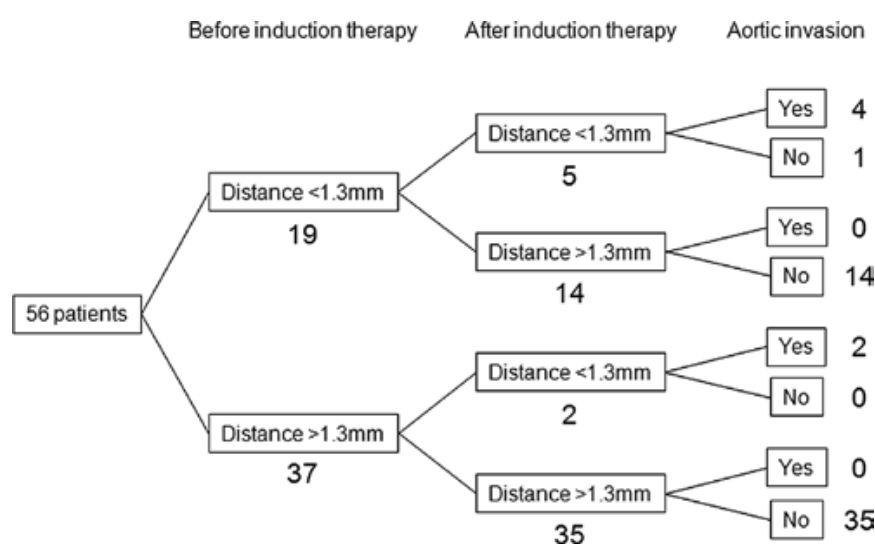

Figure 4. Tumor-to-aorta distances before and after the induction therapy and aortic invasion. When the tumor-to-aorta distance cut-off value was set at $<1.3 \mathrm{~mm}, 19$ patients were suspected to have aortic invasion prior to the induction therapy, 14 of these 19 patients could have a cut-off value of $>1.3 \mathrm{~mm}$ and were eligible to undergo potentially curative resection. Conversely, 2 out of 37 patients had a tumor-to-aorta distance of $<1.3 \mathrm{~mm}$ following the induction therapy. Those who had a tumor-to-aorta distance of $>1.3 \mathrm{~mm}$ prior to the induction therapy were suspected to have progressed tumor invasion of the aortic wall during the induction therapy.

therapy (5). However, there has been significant heterogeneity in the sensitivity and specificity of these modalities (14). In addition, the assessment of tumor size or metabolic status of the tumor does not always reflect the resectability of esophageal cancer.

An intervening fat plane between the esophageal tumor and adjacent structures in the mediastinum accurately indicates a lack of invasion. However, the lack of a fat plane does not necessarily indicate invasion, neither in cachectic patients nor in those of normal body weight (15). Many surgeons have employed the overall circumference of the contact area between the tumor and aortic wall (Picus' angle) to predict aortic invasion in esophageal cancer (10). However, this sign may be considered to be unreliable (3). Indeed, the accuracy of this angle for aortic invasion was only $78.6 \%$, which is inferior to the accuracy of $94.6 \%$ in the tumor-to-aorta distance when the cut-off value was set at $<1.3 \mathrm{~mm}$ in this study.

In the present study, we demonstrated that induction therapy may increase the tumor-to-aorta distance and decrease the maximum tumor size and Picus' angle in each pathological response to induction therapy, which was more evident in patients without aortic invasion. Furthermore, the tumor-to-aorta distance following induction therapy in patients with aortic invasion was significantly reduced compared to patients without aortic invasion. The Picus' angle and minimum CT attenuation value between the tumor and aorta following induction therapy in patients with aortic invasion were significantly greater than in those without aortic invasion. When the tumor-to-aorta distance cut-off value was set at $<1.3 \mathrm{~mm}, 19$ patients were suspected to have aortic invasion prior to the induction therapy, 14 of these 19 patients could have a cut-off value $>1.3 \mathrm{~mm}$ and were eligible to undergo potentially curative resection. Conversely, 2 out of 37 patients had a tumor-to-aorta distance of $<1.3 \mathrm{~mm}$ following the induction therapy; those who had a tumor-to-aorta distance of $>1.3 \mathrm{~mm}$ prior to the induction therapy were suspected to have progressed tumor invasion of the aortic wall during the 
induction therapy. Thus, the evaluation of the tumor-to-aorta distance may be a useful indicator to predict the response to the induction therapy and aortic wall invasion.

The tumor-to-aorta distance following pre-operative chemotherapy was significantly greater than that prior to chemotherapy; however, such a difference was not observed in patients with chemoradiotherapy. This may be due to enhanced inflammation of the connective tissue caused by radiation, which may affect higher CT values.

This study has certain limitations. When the interface between tumor and aorta was not well defined on the pre-treatment study, this method was not applicable. Furthermore, the patients had undergone surgical treatment of esophageal cancer. We selected these patients to enable the exact comparisons of surgical and pathological samples; however, as a result, highly advanced T4 cases were excluded from the study group, which may have increased the number of cases that were almost at the boundary of T3 and T4. Thus, it is necessary to conduct a multicenter, prospective, randomized study in order to verify our current results.

In conclusion, the assessment of the MDCT attenuation value between the esophageal tumor and the aorta is simple, it objectively assesses the response to the induction therapy, and should be a surrogate marker for aortic invasion and the outcome in esophageal cancer. This method should be applied to predict the response to induction therapy, prevent unnecessary surgery in patients with inoperable tumors involving the aortic wall, and to prevent the withholding of curative surgery due to the suggestion of a false-positive result.

\section{Acknowledgements}

The authors greatly thank Yoshihisa Yaguchi, Isao Kumano, Risa Takahata, Kazumichi Yoshida, Hiroyuki Horiguchi and Shinsuke Nomura from the Department of Surgery, National Defense Medical College, for their contribution to data collection and for their critical revision.

\section{References}

1. Entwistle JW III and Goldberg M: Multimodality therapy for resectable cancer of the thoracic esophagus. Ann Thorac Surg 73: 1009-1015, 2002.

2. Gamliel Z and Krasna MJ: Multimodality treatment of esophageal cancer. Surg Clin North Am 85: 621-630, 2005.

3. Diederich S: Staging of oesophageal cancer. Cancer Imaging 7 Spec No A: S63-S66, 2007.

4. Yamabe Y, Kuroki Y, Ishikawa T, Miyakawa K, Kuroki S and Sekiguchi R: Tumor staging of advanced esophageal cancer: combination of double-contrast esophagography and contrast-enhanced CT. Am J Roentgenol 191: 753-757, 2008.

5. Higuchi K, Koizumi W, Tanabe S, et al: Current management of esophageal squamous-cell carcinoma in Japan and other countries. Gastrointest Cancer Res 3: 153-161, 2009.

6. Enzinger PC and Mayer RJ: Esophageal cancer. N Engl J Med 349: 2241-2252, 2003.

7. Gebski V, Burmeister B, Smithers BM, Foo K, Zalcberg J and Simes J: Survival benefits from neoadjuvant chemoradiotherapy or chemotherapy in oesophageal carcinoma: a meta-analysis. Lancet Oncol 8: 226-234, 2007.

8. Chak A, Canto M, Gerdes H, et al: Prognosis of esophageal cancers preoperatively staged to be locally invasive (T4) by endoscopic ultrasound (EUS): a multicenter retrospective cohort study. Gastrointest Endosc 42: 501-506, 1995.

9. Choi J, Kim SG, Kim JS, Jung HC and Song IS: Comparison of endoscopic ultrasonography (EUS), positron emission tomography (PET), and computed tomography (CT) in the preoperative locoregional staging of resectable esophageal cancer. Surg Endosc 24: 1380-1386, 2010.

10. Picus D, Balfe DM, Koehler RE, Roper CL and Owen JW: Computed tomography in the staging of esophageal carcinoma. Radiology 146: 433-438, 1983.

11. Burmeister BH, Walpole ET, D'Arcy N, et al: A phase II trial of chemoradiation therapy with weekly oxaliplatin and protracted infusion of 5-fluorouracil for esophageal cancer. Invest New Drugs 27: 275-279, 2009.

12. Sobin LH and Fleming ID: TNM Classification of Malignant Tumors, 5th edition (1997). Union Internationale Contre le Cancer and the American Joint Committee on Cancer. Cancer 80: 1803-1804, 1997.

13. Japan Esophageal Society: Japanese Classification of Esophageal Cancer, tenth edition: part 1. Esophagus 6: 1-25, 2009.

14. Kwee RM: Prediction of tumor response to neoadjuvant therapy in patients with esophageal cancer with use of $18 \mathrm{~F}$ FDG PET: a systematic review. Radiology 254: 707-717, 2010.

15. Quint LE: Staging work-up of patients with esophageal cancer. Cancer Imaging 7: 128-129, 2007. 